\title{
Dual-console robotic surgery: a new teaching paradigm
}

\author{
Ashlee L. Smith • Eirwen M. Scott • \\ Thomas C. Krivak • Alexander B. Olawaiye • \\ Tianjiao Chu $\cdot$ Scott D. Richard
}

Received: 12 January 2012/Accepted: 19 March 2012/Published online: 4 April 2012

(C) Springer-Verlag London Ltd 2012

\begin{abstract}
Robotic surgery has emerged as an alternative option in minimally invasive gynecologic surgery. The development of the dual-console da Vinci Si Surgical System ${ }^{\circledR}$ has enabled modification of the training atmosphere. We sought to investigate operative times and surgical outcomes while operating with the dual-console model in a training environment for our first fifty cases. We identified the first fifty patients who underwent robot-assisted total hysterectomy (TRH), with or without bilateral salpingooophorectomy (BSO), with or without pelvic and paraaortic lymph node dissection (PPALND), by use of the dual-console robotic system. Records were reviewed for patient demographics and surgical details. All surgery was conducted using the dual-console system and performed by staff physicians and fellows. Operative time was calculated from robotic docking until completion of the procedure. Cases were identified from November 2009 through July 2010. Mean age was 56.2 years (SD 13.35, $95 \%$ CI 52.46-59.86). Mean BMI was 29.5 (SD 7.67, $95 \%$ CI 27.35-31.61). Seventy-eight percent of these patients were considered overweight, including 12 defined as obese (BMI 30-34.9) and 10 patients classified as morbidly obese $(B M I \geq 35)$. Surgery completed included PPALND alone
\end{abstract}

This manuscript was presented during a poster session at the annual meeting of The Western Association of Gynecologic Oncologists, Park City, UT; May 2011.

A. L. Smith $(\bowtie) \cdot$ E. M. Scott · T. C. Krivak ·

A. B. Olawaiye $\cdot$ S. D. Richard

Department of Gynecologic Oncology, Magee-Womens Hospital of UPMC, 300 Halket St., Pittsburgh, PA 15213, USA

e-mail: smitha11@upmc.edu

T. Chu

Magee Womens Research Institute, 204 Craft Ave., Pittsburgh, PA 15213, USA $(n=1)$; radical hysterectomy $(n=1)$; TRH only $(n=3)$; TRH/BSO $(n=25)$; and TRH/BSO/PPALND $(n=20)$. Mean total operating room time was $188.8 \mathrm{~min}$ (SD 55.31, $95 \%$ CI 173.45-204.11). Mean total surgical time for all cases was $118.1 \mathrm{~min}$ (SD 44.28, $95 \%$ CI 105.87-130.41). Two vascular injuries were encountered, with one requiring conversion to laparotomy. These results compare favorably with historically reported outcomes from single-console systems. Utilizing the dual-console enables use of an integrated teaching and supervising environment without compromising operative times or patient outcomes.

Keywords Robotic surgery · Dual-console · Teaching program

\section{Introduction}

Robotic surgery has emerged as an alternative option in minimally invasive gynecologic surgery with increasing penetration into the world of gynecologic oncology. Multiple studies have described the feasibility, efficacy, safety, and adequacy of this approach in managing gynecologic malignancies [1-4]. The da Vinci Surgical System ${ }^{\circledR}$ (Intuitive Surgical, Sunnyvale, CA, USA) has advantages over traditional laparoscopic hysterectomy, including three-dimensional imaging, instruments with wrist-like range of motion, elimination of the fulcrum effect, and faster learning [5]. In a recent survey of Society of Gynecologic Oncology (SGO) members, respondents cited an overall increase in the use of and perceived indications for minimally invasive surgery in the field of gynecologic oncology. In addition, $66 \%$ of physician surveyed planned to increase their use of robotic assisted surgery within the upcoming year [6]. 
Incorporation of robotics into a training environment has been difficult because of the one-surgeon and one-surgery mentality of robotics. Successful training programs have utilized a process involving progressive involvement of additional surgeons, fellows, and residents [7]. Reviews have shown that it can take 20-100 surgeries for a surgeon to reach stable operating times and surgical proficiency when utilizing the robot $[3,8-10]$. With the release of the dual-console da Vinci Si Surgical System ${ }^{\circledR}$ (Intuitive Surgical) in 2009, a redefinition of the training atmosphere for new surgeons has emerged. We sought to investigate outcomes, specifically operative time and surgical outcomes, while operating with the dual-console model in a training environment for our first fifty cases.

\section{Materials and methods}

After obtaining Institutional Review Board approval, we retrospectively identified the first fifty patients who underwent robot-assisted surgery using the dual-console da Vinci Si Surgical System ${ }^{\circledR}$ at Magee-Womens Hospital of UPMC. No patients were excluded from analysis. Decision to perform robotic surgery was left to the discretion of the attending physician and the availability of the robot. This cohort of patients came from all staff physicians at our institution trained in use of the robot (SR, TK, and AO). These physicians were accompanied by the clinical gynecologic oncology fellow assigned to the inpatient service at the time of this review. All patients gave appropriate informed consent before the procedure.

The primary endpoint was total surgical time. Additional endpoints included estimated blood loss (EBL) and complications. Records were reviewed for patients' age, body mass index, pre-operative diagnosis, and procedure. Surgical time was calculated from robotic docking until completion of the procedure. EBL was determined by measurement as documented in the anesthesia records.

All procedures were performed with the dual-console da Vinci Si Surgical System ${ }^{\circledR}$ with two operating surgeons and at least one bedside assistant. Operating surgeons consisted of an attending staff physician and a gynecologic oncology fellow, each at their respective console. A resident physician was used as bedside assistant during these first cases for uterine manipulation, suction/irrigation, and specimen extraction as indicated. Although the staff physicians had completed the required off-site training and proctored cases to be certified for use of the robot, the cases identified in this cohort were the initial cases for each physician. Fellows and resident physicians had only received in-house training before assisting with the surgery.

The robotic surgical technique used is similar to that found on the Intuitive Surgical Instructional website for robotic hysterectomy. Uterine manipulation was accomplished with the V-Care uterine manipulator (Conmed, Utica, NY, USA). Three $8-\mathrm{mm}$ robotic trochars, a $12 \mathrm{~mm}$ camera port, and a $12 \mathrm{~mm}$ bedside assistant port were used. For the purposes of these procedures, the primary surgeon controlled two robotic arms, one on either side of midline. These instruments were the primary operating instruments for the procedure. The second surgeon controlled the third robotic arm and assisted primarily with retraction and manipulation of the uterus with a da Vinci Prograsp. Parts of the surgery were shared between the operating surgeons at each console.

Before surgery all patients underwent mechanical bowel preparation and received appropriate pre-operative antibiotics. DVT prophylaxis consisted of intra-operative pneumatic compression stockings and post-operative Enoxaparin therapy. All patients were admitted after their surgery for inpatient observation. Complications were recorded up to 90 days post-operatively.

Characteristics of the study population and study endpoints were analyzed and described by use of the usual statistics: mean with standard deviation and $95 \%$ confidence intervals (CI). All $95 \%$ CIs for proportions were estimated using the exact binomial distribution. Linear regression analysis was used to examine associations between several variables and case number performed. Cases were identified in the order which they were performed, cases one through fifty.

\section{Results}

Of the fifty patients included in this study, the mean age was 56.2 years (SD 13.35, $95 \%$ CI 52.46-59.86). Mean BMI was 29.5 (SD 7.67, 95 \% CI 27.35-31.61). Seventyeight percent of these patients were considered overweight, including 12 defined as obese (BMI 30-34.9) and 10 classified as morbidly obese (BMI $\geq 35)$. Demographic data are listed in Table 1.

Most of the patients in this cohort were operated on for endometrial cancer $(n=22)$, adnexal mass $(n=12)$, or endometrial hyperplasia $(n=11)$. Remaining pre-operative diagnosis included dysfunctional uterine bleeding $(n=3)$ and cervical cancer $(n=2$; Fig. 1). Forty-six patients underwent a robotic hysterectomy with bilateral salpingo-oophorectomy and three had a robotic hysterectomy alone. Lymphatic staging occurred in $44 \%$ (22/50) of these patients, including one patient who had an interval staging for endometrial cancer. Mean total operating room time (from induction of anesthesia to patient extubation) for the first fifty cases was $188.8 \mathrm{~min}$ (SD 55.31, $95 \% \mathrm{CI}$ 173.45-204.11). Mean total surgical time (from robotic docking to skin incision closure) for all cases was 
Table 1 Pre-operative characteristics

\begin{tabular}{ll}
\hline Age (years) & \\
Mean & 56 \\
Median & 57.5 \\
Range & $22-87$ \\
BMI $\left(\mathrm{kg} / \mathrm{m}^{2}\right)$ & \\
Mean & 30 \\
Median & 27.5 \\
Range & $17-49$ \\
BMI classification $(n)^{\mathrm{a}}$ & \\
Underweight & 3 \\
Normal & 8 \\
Overweight & 17 \\
Obese & 12 \\
Morbidly obese & 10 \\
Race $(n)$ & \\
Caucasian & 49 \\
African American & 1 \\
Pre-operative diagnosis $(n)^{\mathrm{b}}$ & \\
Endometrial cancer & 22 \\
Pelvic mass & 12 \\
Dysplasia & \\
Dysfunctional uterine bleeding & 11 \\
Cervical cancer & 4 \\
\hline
\end{tabular}

${ }^{\mathrm{a}}$ Underweight $=\mathrm{BMI} \quad<20 ; \quad$ Normal $=\mathrm{BMI} \quad 20-24.9 ; \quad$ Overweight $=$ BMI $\quad 25-29.9 ; \quad$ Obese $=$ BMI $\quad 30-34.9 ; \quad$ Morbidly obese $=$ BMI $\geq 35$

b One case was performed for two pre-operative indications. As such, the total number of pre-operative diagnoses is one greater than the total number of cases included in the review

c Cases included those performed for known dysgerminoma, known LMP tumor of the ovary, known ovarian cyst, elevated testosterone suspicious for tumor of ovarian origin, and other uncharacterized pelvic masses

${ }^{d}$ Cases included those performed for endometrial complex atypical hyperplasia, cervical dysplasia, and post-menopausal bleeding

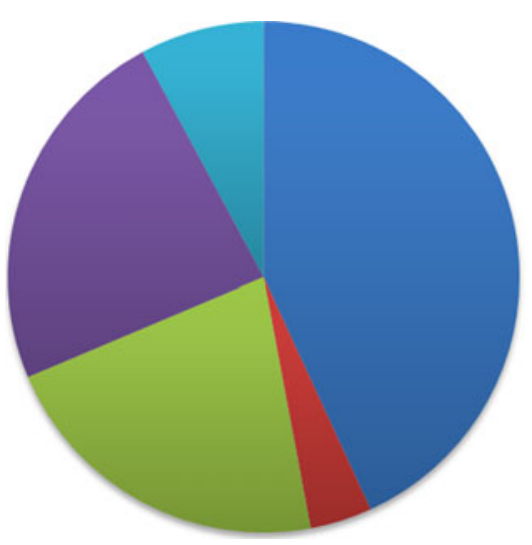

Endometrial Cancer

n Cervical Cancer

Dysplasia

nelvic Mass

- Dysfunctional Uterine Bleeding
$118.1 \mathrm{~min}$ (SD 44.28, $95 \%$ CI 105.87-130.41). When cases were classified as benign or malignant, mean surgical times were 98 min (SD 40.53, $95 \%$ CI 81.43-114.57) and 135 min (SD 40.51, $95 \%$ CI 119.72-150.28), respectively. Mean estimated blood loss was $108.7 \mathrm{ml}$ (SD 98.32, $95 \%$ CI 81.45-135.95; Table 2). Mean number of pelvic and para-aortic lymph nodes removed were 9.3 and 4.6, respectively. Post-operative diagnosis included benign disease, Stage IA, IB, and IIB endometrial cancer, Stage IA ovarian cancer, and Stage IB1 cervical cancer (Fig. 2).

We performed linear regression analysis to study the effect of experience with the robot on total operating room time, total surgical time, LOS, and EBL. Given that the

Table 2 Operative results for 50 patients who underwent gynecologic robot-assisted surgery

Total operating room time (minutes)

Mean 189

SD

55.32

Range

108-314

Total surgical time all cases (minutes)

Mean

118

SD

44.28

Range

$57-251$

Total surgical time benign cases (minutes)

Mean

98

SD

40.53

Range

57-209

Total surgical time malignant cases (minutes)

Mean

SD

Range

$57-251$

Estimated blood loss (cc)

Mean

109

SD

98.32

Range

10-600

Length of stay (days)

Mean $\quad 1.32$

SD $\quad 0.78$

Range 1-4

Total pelvic lymph nodes $(n)$

Mean $\quad 9.35$

SD $\quad 5.12$

Range 2-20

Total para-aortic lymph nodes $(n)$

Mean 4.59

SD $\quad 3.83$

Range 1-14

Fig. 1 Patients' pre-operative diagnosis 


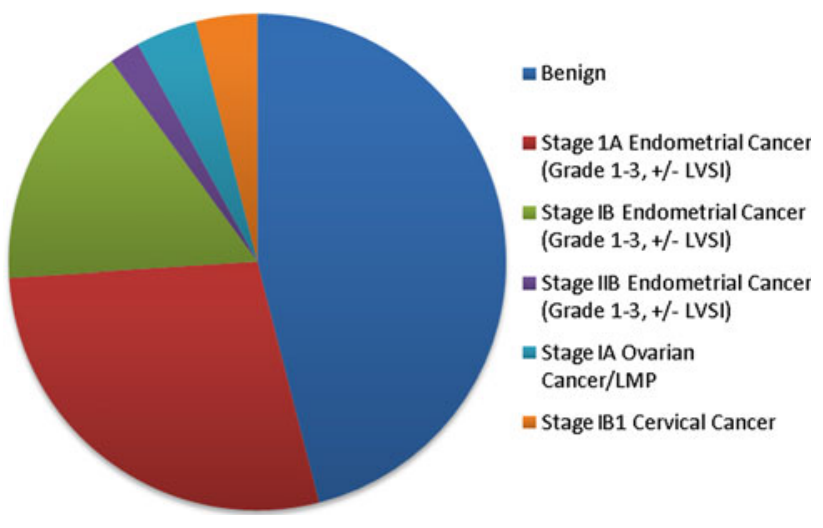

Fig. 2 Post-operative diagnosis by condition and stage

Table 3 Complications

\begin{tabular}{|c|c|}
\hline \\
\hline \\
\hline \multicolumn{2}{|l|}{$\begin{array}{l}\text { Intra-operative complications } \\
\text { Vascular injury } \\
\text { Converted to laparotomy }\end{array}$} \\
\hline \multicolumn{2}{|l|}{ Post-operative complications } \\
\hline \multicolumn{2}{|l|}{ Wound cellulitis } \\
\hline \multicolumn{2}{|l|}{ Vaginal cuff complication ${ }^{\mathrm{b}}$} \\
\hline \multicolumn{2}{|l|}{ Ureteral obstruction } \\
\hline \multicolumn{2}{|l|}{ Pulmonary embolus } \\
\hline Total (excluding 3 return to OR) & \\
\hline
\end{tabular}

data were from our initial incorporation of the dual-console robotic model, cases were examined in the order in which they were performed (ex. 1-50) to obtain an estimate of the progression of our experience with the robot. When controlling for age, BMI, and pre-operative diagnosis, case number is a significant predictor, with negative coefficients for total surgical time and total operating room time ( $p=0.007$ and 0.0018$)$, respectively. Case number was not associated with $\operatorname{LOS}(p=0.3)$ or $\operatorname{EBL}(p=0.56)$. These findings provide evidence that there was improvement in proficiency as operators were familiarized with the techniques and procedures.

Intra-operative complications included two vascular injuries, one of which required conversion to laparotomy for repair of bleeding from the external iliac vein. Neither vascular injury was a direct result of a component of the surgery performed from the dual-console, but rather bleeding encountered during normal lymphatic dissection. Post-operative complications included seven wound infections, two vaginal cuff complications, two ureteral obstructions, and one pulmonary embolism (Table 3). Detection of the first ureteral complication was noted intraoperatively at the time of cystoscopy. Bilateral ureteral jets were noted; however there was a decreased flow on the left.
This patient was managed by urology with ureteral stent placement for four weeks and had no residual problems after stent removal. The second ureteral injury was noted one week post-surgery when the patient returned with flank pain and an abdominal fluid collection. On imaging, extravasation of contrast was noted and ureteral necrosis was identified on a ureteroscope by urology. The patient underwent percutaneous nephrostomy tube placement with subsequent neo-ureterocystostomy six months after the original surgery.

\section{Discussion}

The 21st century brought with it a movement toward minimally invasive surgery in gynecology and gynecologic oncology. The ultimate objective is to maximize those procedures that can be performed safely and accurately via a minimally invasive approach. In 2006, the Gynecologic Oncology Group presented results of a multi-center randomized trial which revealed favorable surgical outcomes when comparing laparoscopy and laparotomy [11, 12]. As more physicians utilized laparoscopic surgery, robotassisted minimally invasive procedures have emerged and gained popularity after approval by the Food and Drug Administration in 2005 for gynecologic procedures $[13,14]$.

There is limited published data on the application of dual-console robotic surgery in gynecology. A recent publication by Marengo et al. [15] reviewed prospective data collected on 33 patients undergoing dual-console robotic surgery for varying indications at their institution. The authors cited a mean operative time of $152 \mathrm{~min}$ and a mean anesthesia time of $196 \mathrm{~min}$. Although a dual-console robotic set-up was used, only one of the surgeons in this review performed the procedure-the other console was used for observation and verbal assistance. The authors did not note a statistically significant difference in operating time between their first 15 and last 18 cases.

Review of additional literature enables us to compare dual-console procedures with the same or similar procedures completed using a single console. The first report of TRH was by Diaz-Arrastia et al. [16]. Data published from this study combined both oncologic and benign procedures. After stratification of their results the authors reported an average EBL of $253 \mathrm{ml}$, average hospital stay of 2.5 days, and overall incidence of complications of $7.3 \%$ when the data from all cancer patients were examined. From 2005 to 2007 Boggess et al. [17] reported an average EBL of $74.5 \mathrm{ml}$, average hospital stay of 1 day, and an average operative time of $191.2 \mathrm{~min}$ for their TRH with staging for endometrial cancer. In comparison, our operative time for staging procedures is significantly less at $135 \mathrm{~min}$. In our 
review, we specifically report our operative times using the dual-console for our first fifty cases, benign and malignant. It should also be noted that at our institution, the dualconsole system is the only system available. Therefore, we are unable to perform an institutional comparison of single versus dual-console surgery.

In 2008 a survey of SGO members was performed by Mabrouk et al. [6], $76 \%$ of respondents reported no or limited laparoscopic training during their fellowship, and $78 \%$ now believe that maximum emphasis should be placed on laparoscopic training. When a similar survey was completed in 2004, only $55 \%$ of respondents noted a high importance of minimally invasive surgery. From this same group surveyed, $24 \%$ indicated they performed robotassisted surgery. Many gynecologic and gynecologic oncology surgeons may utilize the dual-console da Vinci system to overcome the limited experience that clinicians may have faced during their training or earlier parts of their careers.

A major hurdle to success in robotic surgery is the associated learning curve, which applies to both the surgeon and the surgical team. Lenihan et al. [18] demonstrated that the learning curve for benign conditions stabilized at $95 \mathrm{~min}$ after having completed 50 cases. Similar improvements in operative time have been noted in gynecologic oncology; however, none of these reviews evaluated use of the dual-console system [19]. It has been determined that approximately 20-25 surgical robotic cases are required to obtain proficiency using this technique [20, 21]. A recent study by Lim et al. [22] established that learning how to perform robotic surgery at their institution required half the number of cases for proficiency compared with the same cases completed laparoscopically. This could in part be because of the overall increase in exposure of physicians to laparoscopy before adopting robotic surgery. However, the mean operative time in this study for completion of a TRH/PPLAND was $147.2 \pm$ $48.2 \mathrm{~min}$. The mean operative time differed by only 38.1 min when comparing the surgeons' times from before and after their 24th cases. We sought to examine our first 50 cases with reference to the above cited manuscripts which had previously examined proficiency and learning curves for robotic surgery. Our study shows that utilization of the dual-console system gives a second surgeon the opportunity to gain robotic experience, which in turn may result in earlier proficiency.

By improving precision and dexterity, robotic technology enables the surgeon to perform operations that were previously not amenable to minimally invasive surgery. This is especially true for patients that are morbidly obese. Use of the dual-console robotic system at our institution has enabled us to develop and optimize techniques and surgery that are safe, effective, and beneficial to our patients. It has enabled the development of a training module that can be used among physicians with different levels of experience. In our training environment, operating with the dual-console da Vinci $\mathrm{Si}^{\circledR}$ is a safe and feasible option for completion of hysterectomies and staging procedures. A new teaching paradigm has evolved, providing trainees with more exposure and experience in robotic surgeries. Use of the dual-console enables integrated teaching, surgical cooperation with proctoring, and supervision, without compromising operative times or patient outcomes.

Conflict of interest None.

\section{References}

1. Frumovitz M, dos Reis R, Sun CC, Milam MR, Bevers MW, Brown J et al (2007) Comparison of total laparoscopic and abdominal radical hysterectomy for patients with early-stage cervical cancer. Obstet Gynecol 110:96-102

2. Leblanc E, Querleu D, Narducci F, Occelli B, Papageorgiou T, Sonoda Y (2004) Laparoscopic restaging of early stage invasive adnexal tumors: a 10-year experience. Gynecol Oncol 94:624629

3. Abu-Rustum NR, Sonoda Y (2007) Transperitoneal laparoscopic staging with aortic and pelvic lymph node dissection for gynecologic malignancies. Gynecol Oncol 104:5-8

4. Ramirez PT, Slomovitz BM, Soliman PT, Coleman RL, Levenback C (2006) Total laparoscopic radical hysterectomy and lymphadenectomy: the M. D. Anderson Cancer Center experience. Gynecol Oncol 102:252-255

5. Ahlering TE, Skarecky D, Lee D, Clayman RV (2003) Successful transfer of open surgical skills to a laparoscopic environment using a robotic interface: initial experience with laparoscopic radical prostatectomy. J Urol 170:1738-1741

6. Mabrouk M, Frumovitz M, Greer M, Sharma S, Schmeler KM, Soliman PT et al (2009) Trends in laparoscopic and robotic surgery among gynecologic oncologists: a survey update. Gynecol Oncol 112:501-505

7. Mendivil A, Holloway RW, Boggess JF (2009) Emergence of robotic assisted surgery in gynecologic oncology: American perspective. Gynecol Oncol 114:S24-S31

8. Altgassen C, Possover M, Krause N, Plaul K, Michels W, Schneider A (2000) Establishing a new technique of laparoscopic pelvic and para-aortic lymphadenectomy. Obstet Gynecol 95: 348-352

9. Kohler C, Tozzi R, Klemm P, Schneider A (2003) Laparoscopic paraaortic left-sided transperitoneal infrarenal lymphadenectomy in patients with gynecologic malignancies: technique and results. Gynecol Oncol 91:139-148

10. Tozzi R, Malur S, Koehler C, Schneider A (2005) Laparoscopy versus laparotomy in endometrial cancer: first analysis of survival of a randomized prospective study. J Minim Invasive Gynecol 12:130-136

11. Walker JL, Piedmonte MR, Spirtos NM, Eisenkop SM, Schlaerth JB, Mannel RS et al (2009) Laparoscopy compared with laparotomy for comprehensive surgical staging of uterine cancer: Gynecologic Oncology Group Study LAP2. J Clin Oncol 27: 5331-5336

12. Bandera CA, Magrina JF (2009) Robotic surgery in gynecologic oncology. Curr Opin Obstet Gynecol 21:25-30 
13. Magrina JF, Kho RM, Weaver AL, Montero RP, Magtibay PM (2008) Robotic radical hysterectomy: comparison with laparoscopy and laparotomy. Gynecol Oncol 109:86-91

14. Field JB, Benoit MF, Dinh TA, Diaz-Arrastia C (2007) Computer-enhanced robotic surgery in gynecologic oncology. Surg Endosc 21:244-246

15. Marengo F, Larrain D, Babilonti L, Spinillo A (2012) Learning experience using the double-console da Vinci surgical system in gynecology: a prospective cohort study in a university hospital. Arch Gynecol Obstet 285:441-445

16. Diaz-Arrastia C, Jurnalov C, Gomez G, Townsend C Jr (2002) Laparoscopic hysterectomy using a computer-enhanced surgical robot. Surg Endosc 16:1271-1273

17. Boggess JF, Gehrig PA, Cantrell L, Shafer A, Ridgway M, Skinner EN et al. (2008) A comparative study of 3 surgical methods for hysterectomy with staging for endometrial cancer: robotic assistance, laparoscopy, laparotomy. Am J Obstet Gynecol 199:360.e1-360.e9

18. Lenihan JP Jr, Kovanda C, Seshadri-Kreaden U (2008) What is the learning curve for robotic assisted gynecologic surgery? J Minim Invasive Gynecol 15:589-594
19. Sfakianos GP, Frederick PJ, Kendrick JE, Straughn JM, Kilgore LC, Huh WK (2010) Robotic surgery in gynecologic oncology fellowship programs in the USA: a survey of fellows and fellowship directors. Int J Med Robot 6:405-412

20. Lim PC, Kang E, Park do H (2010) Learning curve and surgical outcome for robot-assisted hysterectomy with lymphadenectomy: case-matched controlled comparison with laparoscopy and laparotomy for treatment of endometrial cancer. J Minim Invasive Gynecol 17:739-748

21. Seamon LG, Cohn DE, Richardson DL, Valmadre S, Carlson MJ, Phillips GS et al (2008) Robotic hysterectomy and pelvic-aortic lymphadenectomy for endometrial cancer. Obstet Gynecol 112: 1207-1213

22. Lim PC, Kang E, Park do H (2011) A comparative detail analysis of the learning curve and surgical outcome for robotic hysterectomy with lymphadenectomy versus laparoscopic hysterectomy with lymphadenectomy in treatment of endometrial cancer: a case-matched controlled study of the first one hundred twenty two patients. Gynecol Oncol 120:413-418 